\title{
Finite Element Methods for One Dimensional Fourth Order Semilinear Partial Differential Equation
}

\section{P. Danumjaya ${ }^{1}$}

\begin{abstract}
In this paper, we consider one dimensional fourth order semilinear partial differential equation. Some a priori bounds using Lyapunov functional are derived and existence and uniqueness results for the weak solution are proved. We discuss the finite element Galerkin methods and establish optimal error estimates for the semidiscrete case. Crank-Nicolson scheme is used in the temporal direction and optimal error estimates are derived. Finally, we discuss some numerical experiments and validate with the theoretical results.
\end{abstract}

Keywords Fourth order semilinear partial differential equation - Finite element methods . Extended Fisher-Kolmogorov (EFK) equation - Semidiscrete Galerkin method · Fully discrete Galerkin method · Crank-Nicolson scheme · Optimal error estimates

Mathematics Subject Classification 65L20 - 65L60 - 65L70

\section{Introduction}

We consider the one dimensional second order semilinear partial differential equation:

$$
\frac{\partial u}{\partial t}=\frac{\partial^{2} u}{\partial x^{2}}+u\left(1-u^{2}\right)
$$

The Eq. (1.1) is called as classical Fisher-Kolmogorov (FK) equation and plays an important role in the study of pattern formation in bi-stable systems. The term "bi-stable" refers that $u= \pm 1$ are stable states and $u=0$ is an unstable state.

By adding a stabilizing fourth-order derivative term to the Eq. (1.1), Coullet et al. [4] and by Dee [6] and Van Saarloos [15-17] proposed a modification of (1.1) and obtained the following fourth order semilinear partial differential equation:

\section{P. Danumjaya}

danu@goa.bits-pilani.ac.in

1 Department of Mathematics, BITS Pilani-K K Birla Goa Campus, Sancoale 403726, Goa, India 


$$
\frac{\partial u}{\partial t}=\frac{\partial^{2} u}{\partial x^{2}}-\gamma \frac{\partial^{4} u}{\partial x^{4}}+u-u^{3}
$$

This model is called as the extended Fisher-Kolmogorov (EFK) equation. The Eq. (1.2) arises in a variety of applications such as travelling waves in reaction diffusion systems [1,2], pattern formation in bi-stable systems [6], mezoscopic model of a phase transition in a binary system near the Lipschitz point [7] and propagation of domain walls in liquid crystals [18]. In particular, in the phase transitions near critical points (Lipschitz points), the higher order gradient terms in the free energy functional can no longer be neglected and the fourth order derivative becomes important.

The steady state Eq. of (1.2) has been analysed by Peletier and Troy [11,13] using shooting methods and by Kalies, Kwapisz and Vander Vorst [9] with the help of variational methods. The aim of considering the steady state Eq. of (1.2) is to study the heteroclinic solutions (so called kinks) connecting to the equilibria $u=-1$ and $u=1$. Typically, the stationary problem displays a multitude of periodic, homoclinic and heteroclinic solutions [11,12] depending on the parameter $\gamma$.

The one-dimensional extended Fisher-Kolmogorov (EFK) equation was studied by Danumjaya and Pani [5] by splitting the EFK equation into two second order equations and then analysed by the orthogonal cubic spline collocation (OSC) method. A $H^{1}$-Galerkin mixed finite element method for the EFK equation was studied by Jones and Nandini [8]

In this paper, we study finite element Galerkin methods for the one-dimensional extended Fisher-Kolmogorov (EFK) equation and optimal error estimates are derived in both a semidiscrete and fullydiscrete schemes.

To be more specific, we consider the following initial and boundary value problem: For $\gamma>0$ constant, find a real valued function $u(x, t)$ on $(0, L) \times[0, \infty)$ satisfying

$$
u_{t}+\gamma u_{x x x x}-u_{x x}+f(u)=0, \quad x \in I, t \in(0, \infty)
$$

with initial condition

$$
u(x, 0)=u_{0}(x), \quad x \in I
$$

and boundary conditions

$$
\begin{gathered}
u(0, t)=u(L, t)=0, \quad t \in(0, \infty) \\
u_{x}(0, t)=u_{x}(L, t)=0, \quad t \in(0, \infty) .
\end{gathered}
$$

Here $f(u)=u^{3}-u$, and $I=(0, L)$.

The outline of this article is as follows: In 'Preliminaries' section, some preliminary results and a priori bound using Lyapunov functional are discussed. In 'Semi Discrete Galerkin Method' section, we establish existence uniqueness results for all $t>0$ and optimal error estimates for the semidiscrete Galerkin method are derived. 'Fully Discrete Galerkin Method' section is devoted to fully discrete Galerkin method. Here, we also derive existence uniqueness results and establish optimal error estimates for the backward Euler method and Crank-Nicolson scheme. Finally, in 'Numerical Experiments' section, we discuss some numerical experiments using Crank-Nicolson scheme.

Throughout the paper, $C$ denotes various positive constants which is independent of the discretization parameter $h$ and $k$ which may have different values at different places. 


\section{Preliminaries}

We use the standard notation for the Sobolev spaces and norms. In particular, let $L^{2}(I)$ denotes the space of square integrable function on $I$ and let $(\cdot, \cdot)$ be the natural inner product with induced norm $\|\cdot\|$. For a nonnegative integer $k$, let $H^{k}$ be the Hilbert Sobolev space $H^{k}(I)$ with norm $\|\cdot\|_{k}$.

Let

$$
H_{0}^{2}(I)=\left\{v \in H^{2}(I): v(0)=v(L)=0, v_{x}(0)=v_{x}(L)=0\right\} .
$$

Taking $L^{2}(I)$-inner product of (1.3) with $v \in H_{0}^{2}(I)$, using integration by parts, we obtain the following weak formulation: find $u(\cdot, t) \in H_{0}^{2}(I)$ for $t \in(0, T]$, such that

$$
\begin{aligned}
\left(u_{t}, v\right)+\gamma\left(u_{x x}, v_{x x}\right)+\left(u_{x}, v_{x}\right)+(f(u), v) & =0, \quad \forall v \in H_{0}^{2}(I), \\
u(0) & =u_{0} .
\end{aligned}
$$

For existence of a unique solution to (2.1), the following a priori bound will be useful.

Theorem 2.1 Assume that $u_{0} \in H_{0}^{2}(I)$. Then there exists a constant $C$ such that

$$
\|u(t)\|_{H^{2}(I)} \leq C\left\|u_{0}\right\|_{H^{2}(I)} \text { for } t>0 .
$$

Further,

$$
\|u(t)\|_{W^{1, \infty}(I)} \leq C\left\|u_{0}\right\|_{H^{2}(I)}, \quad t>0 .
$$

Proof We consider the Lyapunov functional $\mathcal{E}(\chi)$ as:

$$
\mathcal{E}(\chi)=\int_{0}^{L}\left\{\frac{\gamma}{2}\left|\chi_{x x}\right|^{2}+\frac{1}{2}\left|\chi_{x}\right|^{2}+F(\chi)\right\} d x,
$$

where $F(\chi)=\frac{1}{4}\left(1-\chi^{2}\right)^{2}$. Note that $F^{\prime}(\chi)=f(\chi)$.

Differentiating (2.2) with respect to $t$, we arrive at

$$
\frac{d \mathcal{E}(u)}{d t}=\int_{0}^{L}\left\{\gamma u_{x x} u_{x x t}+u_{x} u_{x t}+F^{\prime}(u) u_{t}\right\} d x .
$$

Substituting $v=u_{t}$ in (2.1), we obtain

$$
\frac{d \mathcal{E}(u)}{d t}=-\left\|u_{t}\right\|^{2} \leq 0 .
$$

Hence,

$$
\mathcal{E}(u) \leq \mathcal{E}\left(u_{0}\right) .
$$

Finally, we have

$$
\int_{0}^{L}\left\{\frac{\gamma}{2}\left|u_{x x}\right|^{2}+\frac{1}{2}\left|u_{x}\right|^{2}\right\} d x \leq C(\gamma, L)\left\|u_{0}\right\|_{2} .
$$

Thus, using Poincaré inequality, we obtain

$$
\|u(t)\|_{H^{2}(I)} \leq C(\gamma, L)\left\|u_{0}\right\|_{H^{2}(I)}, \quad t>0 .
$$

An appeal to Sobolev imbedding theorem, yields

$$
\|u(t)\|_{W^{1, \infty}(I)} \leq C\left\|u_{0}\right\|_{H^{2}(I)} .
$$

This completes the proof. 


\section{Existence and Uniqueness}

In this subsection, we now discuss existence and uniqueness results.

Theorem 2.2 Let $u_{0} \in H_{0}^{2}(I)$. For $T>0$ there exists $u=u(x, t)$ in $I \times[0, T)$ with

$$
u \in L^{\infty}\left(H_{0}^{2}(I)\right)
$$

and

$$
u_{t} \in L^{\infty}\left(L^{2}(I)\right)
$$

such that $u$ satisfies the initial condition and the Eq. (2.1) in the sense that

$$
\left(u_{t}, \chi\right)+\gamma\left(u_{x x}, \chi_{x x}\right)+\left(u_{x}, \chi_{x}\right)+(f(u), \chi)=0, \quad \forall \chi \in H_{0}^{2}(I) .
$$

Proof Let $\left\{w_{j}\right\}$ be a basis of $H_{0}^{2}(I)$, and let $V_{m}=\operatorname{span}\left\{w_{1}, w_{2}, \ldots, w_{m}\right\}$. Define for each $t>0$

$$
u_{m}(t)=\sum_{i=1}^{m} g_{i m}(t) w_{i} \in V_{m}
$$

as a solution of

$$
\begin{aligned}
\left(u_{m t}, w_{j}\right)+\gamma\left(u_{m}^{\prime \prime}, w_{j}^{\prime \prime}\right)+\left(u_{m}^{\prime}, w_{j}^{\prime}\right)+\left(f\left(u_{m}\right), w_{j}\right) & =0, \quad j=1,2, \ldots, m \\
u_{m}(0) & =u_{0, m},
\end{aligned}
$$

where $u_{0, m}=u_{m}(0)=\sum_{i=1}^{m} g_{i m} w_{i} \rightarrow u_{0}$ in $H_{0}^{2}(I)$. Now (2.4) leads to a system of nonlinear ODE's. Then an application of Picard's theorem ensures the existence of a solution $u_{m}$ to (2.4) which is valid in $\left[0, t_{m}\right]$, for some $t_{m}>0$. In order to prove global existence, we need the following a priori bounds.

Letting the Lyapunov functional $\mathcal{E}(\cdot)$ as

$$
\mathcal{E}\left(u_{m}\right)=\int_{0}^{L}\left\{\frac{\gamma}{2}\left|u_{m}^{\prime \prime}\right|^{2}+\frac{1}{2}\left|u_{m}^{\prime}\right|^{2}+F\left(u_{m}\right)\right\} d x,
$$

where $F\left(u_{m}\right)=\frac{1}{4}\left(1-u_{m}\right)^{2}$, we obtain as in the case of Theorem 2.1, we arrive at

$$
\mathcal{E}\left(u_{m}\right) \leq \mathcal{E}\left(u_{0, m}\right),
$$

since $\left\|u_{0, m}\right\|_{2} \leq C$, we obtain the following estimates

$$
\left\|u_{m}(t)\right\|,\left\|u_{m}^{\prime}(t)\right\|,\left\|u_{m}^{\prime \prime}(t)\right\| \leq C \text { (independent of } \mathrm{m} \text { and } \mathrm{t} \text { ). }
$$

Therefore,

$$
\begin{aligned}
& u_{m} \text { is bounded in } L^{\infty}\left(H^{2}(I)\right), \\
& u_{m}^{\prime \prime} \text { is bounded in } L^{\infty}\left(L^{2}(I)\right) .
\end{aligned}
$$

Using the boundedness of $\left\|u_{m}\right\|_{L^{\infty}\left(H^{2}\right)}$, we obtain

$$
\left\|f\left(u_{m}\right)\right\|^{2}=\int_{0}^{L}\left(u_{m}^{3}-u_{m}\right)^{2} d x \leq 2 \int_{0}^{L} u_{m}^{6} d x+2 \int_{0}^{L} u_{m}^{2} d x \leq C,
$$

and hence,

$$
f\left(u_{m}\right) \text { is bounded in } L^{\infty}\left(L^{2}(I)\right) .
$$


Now let $j$ be fixed and $m>j$,

$$
\begin{aligned}
& \left(u_{m}^{\prime \prime}, w_{j}^{\prime \prime}\right) \rightarrow\left(u^{\prime \prime}, w_{j}^{\prime \prime}\right) \text { in } L^{\infty}(0, T) \text { weak*, } \\
& \left(u_{m}^{\prime}, w_{j}^{\prime}\right) \rightarrow\left(u^{\prime}, w_{j}^{\prime}\right) \text { in } L^{\infty}(0, T) \text { weak*, } \\
& \left(f\left(u_{m}\right), w_{j}\right) \rightarrow\left(f(u), w_{j}\right) \text { in } L^{\infty}(0, T) \text { weak*. }
\end{aligned}
$$

Also, since

$$
\left(u_{m t}, w_{j}\right) \rightarrow\left(u_{t}, w_{j}\right) \text { in } \mathcal{D}^{\prime}(0, T)
$$

We obtain

$$
\left(u_{t}, w_{j}\right)+\gamma\left(u^{\prime \prime}, w_{j}\right)+\left(u^{\prime}, w_{j}\right)+\left(f(u), w_{j}\right)=0 .
$$

The Eq. (2.3) follows from the denseness of the basis $\left\{w_{j}\right\}$ in $H_{0}^{2}(I)$.

Uniqueness Suppose $u$ and $v$ are two solutions of (2.3).

Taking $w=u-v$, we obtain

$$
\left(w_{t}, \chi\right)+\gamma\left(w_{x x}, \chi_{x x}\right)+\left(w_{x}, \chi_{x}\right)=-(f(u)-f(v), \chi)
$$

Setting $\chi=w$ and using the boundedness of $\|u\|_{\infty}$ and $\|v\|_{\infty}$, we obtain

$$
\|w(t)\|^{2} \leq\|w(0)\|^{2}+C \int_{0}^{t}\|w(\tau)\|^{2} d \tau .
$$

An application of Gronwall's lemma yields

$$
\|w(t)\|^{2} \leq\|w(0)\|^{2} \exp (C T) .
$$

Using $w(0)=u(0)-v(0)=u_{0}-u_{0}=0$, we obtain $w(t)=0$. Thus, $u=v$ and this completes the uniqueness of the solution.

\section{Semi Discrete Galerkin Method}

In this section, we discuss the semidiscrete Galerkin method for the EFK Eqs. (1.3)-(1.5) and derive optimal error estimates.

\section{Galerkin Approximation}

Let $S_{h}^{0}$ be a finite dimensional subspace of $H_{0}^{2}(I)$ with the following approximation property:

For $v \in H_{0}^{2}(I) \cap W^{s, p}(I)$, there exists a constant $C$ independent of $h$ such that

$$
\inf _{\chi \in S_{h}^{0}} \sum_{j=0}^{2} h^{j}\left\|D^{j}(v-\chi)\right\|_{L^{p}} \leq C h^{s}\|v\|_{W^{s, p}(I)}, \quad 2 \leq s \leq r+1, \quad 1 \leq p \leq \infty .
$$

Moreover, assume that $S_{h}^{0}$ satisfies the following inverse hypothesis: for $\chi \in S_{h}^{0}$

$$
\|\chi\|_{W^{j, \infty}(I)} \leq C h^{-1 / 2}\|\chi\|_{H^{j}(I)}, \quad j=0,1 .
$$

To be more specific, let $\left\{x_{0}<x_{1}<x_{2}<\cdots<x_{N}\right\}$ be a partition of $I$ with

$$
I_{i}=\left(x_{i-1}, x_{i}\right), h_{i}=x_{i}-x_{i-1} \text {, and } h=\max _{1 \leq i \leq N} h_{i} .
$$


Let

$$
\begin{gathered}
S_{h}^{0}=\left\{v \in C^{1}(0, L):\left.v\right|_{I_{i}} \in P_{r}\left(I_{i}\right), i=1,2, \ldots, N\right. \\
\text { and } \left.v(0)=v(L)=0, v^{\prime}(0)=v^{\prime}(L)=0\right\},
\end{gathered}
$$

where $P_{r}$ is a space of polynomial of degree $\leq r$ with $r \geq 2$. It is easy to check that the space $S_{h}^{0}$ satisfies the above approximation and inverse properties provided the partition is quasi-uniform. Examples of such spaces can be found in [3].

The semidiscrete Galerkin approximation of (1.3) is defined to be a function $u_{h}:(0, T] \rightarrow$ $S_{h}^{0}$ such that

$$
\begin{aligned}
&\left(u_{h t}, \chi\right)+\gamma\left(u_{h x x}, u_{x x}\right)+\left(u_{h x}, \chi_{x}\right)+\left(f\left(u_{h}\right), \chi\right)=0, \quad \forall \chi \in S_{h}^{0} \\
& \text { with } u_{h}(0)=u_{0, h},
\end{aligned}
$$

where $u_{0, h}$ is an appropriate approximation of $u_{0}$ in $S_{h}^{0}$ to be defined later.

For the wellposedness of (3.1), we use the fact that $S_{h}^{0}$ is a finite dimensional subspace and let $\left\{\varphi_{i}\right\}_{i=1}^{N_{h}}$ be a basis of $S_{h}^{0}$. We now express the solution $u_{h}$ as finite linear combination of basis functions as

$$
u_{h}(x, t)=\sum_{i=1}^{N_{h}} \alpha_{i}(t) \varphi_{i}(x) .
$$

Setting $\chi=\varphi_{j}$ in (3.1) and using (3.2), we arrive at

$$
\sum_{i=1}^{N_{h}} \alpha_{i}^{\prime}(t)\left(\varphi_{i}, \varphi_{j}\right)+\sum_{i=1}^{N_{h}} \alpha_{i}(t)\left(\gamma\left(\varphi_{i}^{\prime \prime}, \varphi_{j}^{\prime \prime}\right)+\left(\varphi_{i}^{\prime}, \varphi_{j}^{\prime}\right)\right)=-\left(f\left(\sum_{i=1}^{N_{h}} \alpha_{i} \varphi_{i}\right), \varphi_{j}\right) .
$$

In matrix notation, we express as

$$
A \alpha^{\prime}(t)+B \alpha=F(\alpha)
$$

where $A=\left[a_{i j}\right]$ is the mass matrix with elements $a_{i j}=\left(\varphi_{i}, \varphi_{j}\right), B=\left[b_{i j}\right]$ is the stiffness matrix with $b_{i j}=\gamma\left(\varphi_{i}^{\prime \prime}, \varphi_{j}^{\prime \prime}\right)+\left(\varphi_{i}^{\prime}, \varphi_{j}^{\prime}\right)$, and $F_{j}(\alpha)=-\left(f\left(\sum \alpha_{i} \varphi_{i}\right), \varphi_{j}\right)$. Since all $\varphi_{i}$ 's are linearly independent, $A$ is invertible. Thus, we have a system of nonlinear ordinary differential equations

$$
\alpha^{\prime}(t)+A^{-1} B \alpha(t)=A^{-1} F(\alpha), \quad t>0 .
$$

From Picard's theorem, there exists a positive time $t^{*}>0$ such that (3.3) has a unique solution $\alpha$ and hence, $u_{h}$ in $\left[0, t^{*}\right)$. For proving the global existence, we need an a priori bound like $\left\|u_{h}(t)\right\|_{L^{\infty}\left(H^{2}\right)}<C$. Then using continuation argument, it is easy to show the existence of a unique solution $u_{h}$ to (3.1) for all $t>0$.

As in the case of continuous problem, we again use Lyapunov functional $\mathcal{E}\left(u_{h}\right)$ to derive the following a priori bound:

$$
\left\|u_{h}(t)\right\|_{W^{j, \infty}} \leq C\left\|u_{h}(t)\right\|_{2} \leq C\left\|u_{0, h}\right\|_{2}, \quad j=0,1 .
$$

\section{Error Estimates}

In this sub-section, we discuss optimal error estimates for the semidiscrete scheme.

Very often a direct comparison between $u$ and $u_{h}$ does not yields optimal error estimates. Therefore, there is a need to introduce an appropriate auxiliary function $\tilde{u}_{h}$. We split the error as 


$$
u-u_{h}=\left(u-\tilde{u}_{h}\right)-\left(u_{h}-\tilde{u}_{h}\right)=\eta-\theta .
$$

We introduce the bilinear form:

$$
A(v, w)=\gamma\left(v_{x x}, w_{x x}\right)+\left(v_{x}, w_{x}\right), \quad \forall v, w \in H_{0}^{2}(I) .
$$

Note that $A(\cdot, \cdot)$ satisfies the following properties :

(i) Boundedness There is a positive constant $M$ such that

$$
|A(v, w)| \leq M\|v\|_{2}\|w\|_{2} \quad \forall v, w \in H_{0}^{2}(I) .
$$

(ii) Coercivity There is a constant $\alpha_{0}>0$ such that

$$
A(v, v) \geq \alpha_{0}\|v\|_{2}^{2} \quad \forall v \in H_{0}^{2}(I) .
$$

Let $\tilde{u}_{h}$ be an auxiliary projection of $u$ defined by

$$
A\left(u-\tilde{u}_{h}, \chi\right)=0, \quad \forall \chi \in S_{h}^{0} .
$$

Setting $\eta=u-\tilde{u}_{h}$, then the following estimates are easy to obtain. For a proof, we refer $[3]$.

$$
\begin{gathered}
\|\eta\|_{j}+\left\|\eta_{t}\right\|_{j} \leq C h^{r+1-j}\left(\sum_{m=0}^{1}\left\|\frac{\partial^{m} u}{\partial t^{m}}\right\|_{r+1}\right) \quad j=0,1,2 \\
\|\eta\|_{W^{j}, \infty} \leq C h^{r+1-j}\|u\|_{W^{r+1, \infty}(I)}, \quad j=0,1 .
\end{gathered}
$$

We subtract the Eq. (3.1) from (2.1) and using the auxiliary projection (3.5), we obtain the error equation in $\theta$ as

$$
\left(\theta_{t}, \chi\right)+A(\theta, \chi)=\left(\eta_{t}, \chi\right)+\left(f(u)-f\left(u_{h}\right), \chi\right), \quad \forall \chi \in S_{h}^{0} .
$$

Theorem 3.1 Let $u$ and $u_{h}$ be the solutions of (2.1) and (3.1), respectively, and let $u_{h}(0)=$ $\tilde{u}_{0, h}$. Then

$$
\left\|u(t)-u_{h}(t)\right\| \leq C(T) h^{r+1}\left[\|u\|_{L^{\infty}\left(H^{r+1}\right)}+\left\|u_{t}\right\|_{L^{2}\left(H^{r+1}\right)}\right] .
$$

Proof Since we know the estimates of $\eta$, we now find $\theta$ estimates and then using the triangle inequality, we find the error estimates.

Setting $\chi=\theta$ in (3.8), we obtain

$$
\left(\theta_{t}, \theta\right)+A(\theta, \theta)=\left(\eta_{t}, \theta\right)+\left(f(u)-f\left(u_{h}\right), \theta\right) .
$$

A use of coercivity of $A(\cdot, \cdot)$ yields

$$
\frac{1}{2} \frac{d}{d t}\|\theta\|^{2}+\alpha\|\theta\|_{2}^{2} \leq\left(\eta_{t}, \theta\right)+\left(f(u)-f\left(u_{h}\right), \theta\right) .
$$

To estimate the last term in the right hand side of (3.9), we rewrite it as

$$
\begin{aligned}
\left(\left(u^{3}-u\right)-\left(u_{h}^{3}-u_{h}\right), \theta\right) & =\left(\left(u-u_{h}\right)\left(u^{2}+u u_{h}+u_{h}^{2}-1\right), \theta\right) \\
& =\left((\eta-\theta)\left(u^{2}+u u_{h}+u_{h}^{2}-1\right), \theta\right) .
\end{aligned}
$$

Using the $L^{\infty}$-bounds for $u, u_{h}$ and applying Cauchy Swarz inequality as well as Young's inequality, we find

$$
\left|\left(f(u)-f\left(u_{h}\right), \theta\right)\right| \leq C\left(\|\eta\|^{2}+\|\theta\|^{2}\right) .
$$


Integration with respect to $t$, we obtain

$$
\begin{aligned}
\|\theta(t)\|^{2}+2 \alpha \int_{0}^{t}\|\theta(s)\|^{2} d s & \leq\|\theta(0)\|^{2}+2 \int_{0}^{t}\left\|\eta_{t}(s)\right\|\|\theta(s)\| d s \\
& +C \int_{0}^{t}\left(\|\eta(s)\|^{2}+\|\theta(s)\|^{2}\right) d s .
\end{aligned}
$$

Since $\theta(0)=0$, it follows that

$$
\|\theta(t)\|^{2} \leq C \int_{0}^{t}\left(\left\|\eta_{t}(s)\right\|^{2}+\|\eta(s)\|^{2}\right) d s+C_{1} \int_{0}^{t}\|\theta(s)\|^{2} d s .
$$

Using the estimates of $\eta, \eta_{t}$ from (3.6)-(3.7) and an application of Gronwall's inequality yields

$$
\|\theta(t)\|^{2} \leq C h^{2(r+1)}\left(\|u\|_{L^{2}\left(H^{r+1}\right)}^{2}+\left\|u_{t}\right\|_{L^{2}\left(H^{r+1}\right)}^{2}\right) \exp \left(C_{1} T\right) .
$$

The result follows using triangle inequality and this completes the proof of the theorem.

Remark Note that instead of function $u_{0, h}=\tilde{u}_{0}$, we can use $u_{0, h}$ as an interpolant of $u_{0}$ onto $S_{h}^{0}$.

Theorem 3.2 Let $u$ and $u_{h}$ be the solutions of (2.1) and (3.1), respectively, and let $u_{h}(0)=$ $\tilde{u}_{0, h}$. Then

$$
\left\|u(t)-u_{h}(t)\right\|_{j} \leq C h^{r+1-j}\left[\|u\|_{L^{\infty}\left(H^{r+1}\right)}+\left\|u_{t}\right\|_{L^{2}\left(H^{r+1}\right)}\right], \quad j=1,2 .
$$

Moreover,

$$
\left\|u(t)-u_{h}(t)\right\|_{W^{j, \infty}} \leq C h^{r+1-j}\left[\|u\|_{L^{\infty}\left(W^{r+1, \infty}\right)}+\left\|u_{t}\right\|_{L^{2}\left(H^{r+1}\right)}\right], \quad j=0,1 .
$$

Proof Setting $\chi=\theta_{t}$ in (3.8), we obtain

$$
\left\|\theta_{t}\right\|^{2}+\frac{1}{2} \frac{d}{d t}\left(\gamma\left\|\theta_{x x}\right\|^{2}+\left\|\theta_{x}\right\|^{2}\right)=\left(\eta_{t}, \theta_{t}\right)+\left(f(u)-f\left(u_{h}\right), \theta_{t}\right) .
$$

Integrating with respect to $t$, it follows that

$$
\begin{aligned}
2 \int_{0}^{t}\left\|\theta_{t}(s)\right\|^{2} d s+\gamma\left\|\theta_{x x}\right\|^{2}+\left\|\theta_{x}\right\|^{2} \leq & 2 \int_{0}^{t}\left\|\eta_{t}(s)\right\|\left\|\theta_{t}(s)\right\| d s \\
& +2 \int_{0}^{t}\|\eta-\theta\|\left\|u^{2}+u u_{h}+u_{h}^{2}-1\right\|\left\|\theta_{t}(s)\right\| d s .
\end{aligned}
$$

Using the $L^{\infty}$-bounds for $u$ and $u_{h}$, we arrive at

$$
\begin{aligned}
& 2 \int_{0}^{t}\left\|\theta_{t}(s)\right\|^{2} d s+\gamma\left\|\theta_{x x}\right\|^{2}+\left\|\theta_{x}\right\|^{2} \\
& \quad \leq 2 \int_{0}^{t}\left\|\eta_{t}(s)\right\|\left\|\theta_{t}(s)\right\| d s+2 C \int_{0}^{t}\|\eta(s)\|\left\|\theta_{t}(s)\right\| d s+2 C \int_{0}^{t}\|\theta(s)\|\left\|\theta_{t}(s)\right\| d s .
\end{aligned}
$$


We use Young's inequality, we obtain

$$
\begin{aligned}
& 2 \int_{0}^{t}\left\|\theta_{t}(s)\right\|^{2} d s+\gamma\left\|\theta_{x x}\right\|^{2}+\left\|\theta_{x}\right\|^{2} \\
& \leq 2 \int_{0}^{t}\left\|\eta_{t}(s)\right\|^{2} d s+\frac{1}{2} \int_{0}^{t}\left\|\theta_{t}(s)\right\|^{2} d s+C\left(\int_{0}^{t}\|\eta(s)\|^{2}+\|\theta(s)\|^{2}\right) d s \\
& \quad+\int_{0}^{t}\left\|\theta_{t}(s)\right\|^{2} d s .
\end{aligned}
$$

Hence, using Poincaré inequality, we obtain

$$
\|\theta(t)\|_{H^{2}}^{2} \leq \int_{0}^{t}\left\|\eta_{t}(s)\right\|^{2} d s+2 C \int_{0}^{t}\left(\|\eta(s)\|^{2}+\|\theta(s)\|^{2}\right) d s .
$$

Substituting $\eta, \eta_{t}$ estimates from (3.6)-(3.7), and $\theta$ estimate in (3.11), we find that

$$
\|\theta(t)\|_{H^{2}}^{2} \leq C h^{2(r+1)}\left(\|u(t)\|_{L^{2}\left(H^{r+1}\right)}^{2}+\left\|u_{t}\right\|_{L^{2}\left(H^{r+1}\right)}^{2}\right) .
$$

A use of Sobolev imbedding theorem, we obtain

$$
\|\theta(t)\|_{W^{j, \infty}}^{2} \leq C h^{2(r+1)}\left(\|u(t)\|_{L^{2}\left(H^{r+1}\right)}^{2}+\left\|u_{t}\right\|_{L^{2}\left(H^{r+1}\right)}^{2}\right), \quad j=0,1 .
$$

Using triangle inequality, we complete the rest of the proof.

\section{Fully Discrete Galerkin Method}

In this section, we discuss the discrete-time Galerkin approximations and derive some $a$ priori bounds.

\section{Crank-Nicolson Scheme}

For the second order accuracy in time, we now consider the Crank-Nicolson scheme.

For $\varphi \in C[0, T]$, let

$$
\bar{\partial}_{t} \varphi^{n}=\frac{\varphi^{n}-\varphi^{n-1}}{k}, \quad \varphi^{n-1 / 2}=\frac{\varphi^{n}+\varphi^{n-1}}{2} .
$$

Following Qiang and Nicolaides [14], we define

$$
\tilde{f}\left(U^{n-1}, U^{n}\right)= \begin{cases}\frac{F\left(U^{n-1}\right)-F\left(U^{n}\right)}{U^{n-1}-U^{n}}, & U^{n-1} \neq U^{n} \\ F^{\prime}\left(U^{n-1}\right), & U^{n-1}=U^{n} .\end{cases}
$$

The discrete time Galerkin approximation $U^{n}$ of $u\left(t_{n}\right)$ is defined as a solution of

$$
\begin{aligned}
\left(\bar{\partial}_{t} U^{n}, \chi\right)+\gamma\left(U_{x x}^{n-1 / 2}, \chi_{x x}\right) & +\left(U_{x}^{n-1 / 2}, \chi_{x}\right) \\
& +\left(\tilde{f}\left(U^{n-1}, U^{n}\right), \chi\right)=0, \quad \forall \chi \in S_{h}^{0} \\
U^{0} & =u_{0, h},
\end{aligned}
$$

where, $u_{0, h} \in S_{h}^{0}$ is an appropriate approximation to $u_{0}$ to be defined later.

The following a priori bounds are useful for proving the optimal error estimates. 
Theorem 4.1 Let $U^{n}$ be a solution of (4.1). Then there exists a positive constant $C$ such that

$$
\left\|U^{n}\right\|_{2} \leq C\left\|U^{0}\right\|_{2}, \quad n \geq 1 .
$$

Proof Choose $\chi=U^{n}-U^{n-1}$ in (4.1), we obtain

$$
\begin{aligned}
& \frac{1}{k}\left\|U^{n}-U^{n-1}\right\|^{2}+\frac{\gamma}{2}\left(\left\|U_{x x}^{n}\right\|^{2}-\left\|U_{x x}^{n}\right\|^{2}\right)+\frac{1}{2}\left(\left\|U_{x}^{n}\right\|^{2}-\left\|U_{x}^{n-1}\right\|^{2}\right) \\
& \quad+\left(F\left(U^{n}\right)-F\left(U^{n-1}\right), 1\right)=0 .
\end{aligned}
$$

Using the definition of $\mathcal{E}(\cdot)$, we note that

$$
\begin{aligned}
\mathcal{E}\left(U^{n}\right)-\mathcal{E}\left(U^{n-1}\right)= & \frac{\gamma}{2}\left(\left\|U_{x x}^{n}\right\|^{2}-\left\|U_{x x}^{n-1}\right\|^{2}\right)+\frac{1}{2}\left(\left\|U_{x}^{n}\right\|^{2}-\left\|U_{x}^{n-1}\right\|^{2}\right) \\
& +\left(F\left(U^{n}\right)-F\left(U^{n-1}\right), 1\right) .
\end{aligned}
$$

Using (4.2) in (4.4), we obtain

$$
\mathcal{E}\left(U^{n}\right)-\mathcal{E}\left(U^{n-1}\right)=-\frac{1}{k}\left\|U^{n}-U^{n-1}\right\|^{2} .
$$

and hence

$$
\mathcal{E}\left(U^{n}\right) \leq \mathcal{E}\left(U^{n-1}\right)
$$

Recursively, we find that

$$
\mathcal{E}\left(U^{n}\right) \leq \cdots \leq \mathcal{E}\left(U^{0}\right) .
$$

From the definition of $\mathcal{E}\left(U^{n}\right)$, it follows that

$$
\int_{0}^{L}\left\{\left.\frac{\gamma}{2}\left|U_{x x}^{n} \|^{2}+\frac{1}{2}\right| U_{x}^{n}\right|^{2}+F\left(U^{n}\right)\right\} d x \leq \int_{0}^{L}\left\{\frac{\gamma}{2}\left|U_{0, x x}^{n}\right|^{2}+\frac{1}{2}\left|U_{x}^{n}\right|^{2}+F\left(U_{0}^{n}\right)\right\} d x .
$$

As $F\left(U^{n}\right)=\frac{1}{4}\left(1-\left(U^{n}\right)^{2}\right)^{2} \geq 0$, we obtain

$$
\left\|U^{n}\right\|_{2} \leq C\left\|U^{0}\right\|_{2} .
$$

A use of Sobolev imbedding theorem yields

$$
\left\|U^{n}\right\|_{W^{j, \infty}} \leq C\left\|U^{0}\right\|_{2} . \quad j=0,1 .
$$

\section{Error Estimates}

We discuss the optimal error estimates for the Crank-Nicolson scheme.

The error equation in $\theta^{n}$ is

$$
\begin{aligned}
& \left(\bar{\partial}_{t} \theta^{n}, \chi\right)+\gamma\left(\theta_{x x}^{n-1 / 2}, \chi_{x x}\right)+\left(\theta_{x}^{n-1 / 2}, \chi_{x}\right) \\
& \quad=\left(f\left(u^{n-1 / 2}\right)-\tilde{f}\left(U^{n-1 / 2}\right), \chi\right)+\left(\sigma^{n}, \chi\right)-\left(\bar{\partial}_{t} \eta^{n}, \chi\right)
\end{aligned}
$$

where

$$
\sigma^{n}=u_{t}^{n-1 / 2}-\bar{\partial}_{t} u\left(t_{n}\right)
$$


Theorem 4.2 Let $U^{n}$ and $u$ be the solutions of (4.1) and (2.1), respectively, and let $U^{0}=\tilde{u}_{0, h}$. Then, there is a positive constant $C$ such that

$\left\|U^{n}-u\left(t_{n}\right)\right\| \leq C h^{r+1}\left\{\|u\|_{L^{\infty}\left(H^{r+1}\right)}+\int_{0}^{t_{n}}\left\|u_{t}\right\|_{L^{2}\left(H^{r+1}\right)} d s\right\}+k^{2} \int_{0}^{t_{n}}\left\|u_{t t t}\right\| d s, \quad n \geq 0$.

Proof Setting $\chi=\theta^{n-1 / 2}$, in (4.4), we obtain

$$
\begin{aligned}
& \frac{1}{2 k}\left(\left\|\theta^{n}\right\|^{2}-\left\|\theta^{n-1}\right\|^{2}\right)+\gamma\left\|\theta_{x x}^{n-1 / 2}\right\|^{2}+\left\|\theta_{x}^{n-1 / 2}\right\|^{2} \\
& \quad=\left(f\left(u^{n-1 / 2}\right)-\tilde{f}\left(U^{n-1 / 2}\right), \theta^{n-1 / 2}\right)+\left(\sigma^{n}, \theta^{n-1 / 2}\right)-\left(\bar{\partial}_{t} \eta^{n}, \theta^{n-1 / 2}\right) .
\end{aligned}
$$

This implies that

$$
\begin{aligned}
\frac{1}{2 k}\left(\left\|\theta^{n}\right\|^{2}-\left\|\theta^{n-1}\right\|^{2}\right) & \leq\left(f\left(u^{n-1 / 2}\right)-\tilde{f}\left(U^{n-1 / 2}\right), \theta^{n-1 / 2}\right) \\
& +\left(\sigma^{n}, \theta^{n-1 / 2}\right) .
\end{aligned}
$$

Using the boundedness of $\|u\|$ and $\left\|U^{n}\right\|$, We find

$$
\left(f\left(u^{n-1 / 2}\right)-f\left(U^{n-1 / 2}\right), \theta^{n-1 / 2}\right) \leq C\left(\left\|\theta^{n-1 / 2}\right\|^{2}+\left\|\eta^{n-1 / 2}\right\|^{2}\right) .
$$

Thus, we obtain

$$
\left\|\theta^{n}\right\|^{2} \leq C k\left(\left\|\theta^{n-1 / 2}\right\|^{2}+\left\|\eta^{n-1 / 2}\right\|^{2}+\left\|\sigma^{n}\right\|^{2}+\left\|\bar{\partial}_{t} \eta^{n}\right\|^{2}\right) .
$$

Using $\theta^{n-1 / 2}=\frac{\theta^{n}+\theta^{n-1}}{2}$ and simplifying, we obtain

$$
\left\|\theta^{n}\right\|^{2} \leq C k\left(\left\|\theta^{n-1}\right\|^{2}+\left\|\eta^{n-1 / 2}\right\|^{2}+\left\|\sigma^{n}\right\|^{2}+\left\|\bar{\partial}_{t} \eta^{n}\right\|^{2}\right) .
$$

We note that

$$
\left\|\sigma^{n}\right\|^{2} \leq C k^{3} \int_{t_{n-1}}^{t_{n}}\left\|u_{t t t}(s)\right\|^{2} d s .
$$

Substituting $\sigma^{n}, \bar{\partial}_{t} \eta^{n}$ and $\eta^{n}$ values in (4.6), we obtain the required result.

\section{Numerical Experiments}

In this section, we show some numerical experiments for the one-dimensional EFK equation.

We discuss the computational experiments for the following one dimensional EFK equation using cubic Hermite basis functions.

Table 1 The order of convergence for $u(x, t)$ at $t=1.0$ and $\gamma=0.01$

\begin{tabular}{lllll}
\hline $\mathrm{N}$ & $\left\|u_{h}-u_{h_{i}}\right\|_{L^{\infty}}$ & Order & $\left\|u_{h}-u_{h_{i}}\right\|_{L^{2}}$ & Order \\
\hline 20 & $2.4512 \times 10^{-7}$ & & $7.8607 \times 10^{-7}$ & \\
40 & $3.5273 \times 10^{-8}$ & 2.7968 & $1.1342 \times 10^{-7}$ & 2.7930 \\
80 & $3.1303 \times 10^{-9}$ & 3.4942 & $1.0022 \times 10^{-8}$ & 3.5004 \\
160 & $2.1704 \times 10^{-10}$ & 3.8503 & $6.9548 \times 10^{-10}$ & 3.8490 \\
320 & $1.2975 \times 10^{-11}$ & 4.0641 & $4.1584 \times 10^{-11}$ & 4.0639 \\
\hline
\end{tabular}




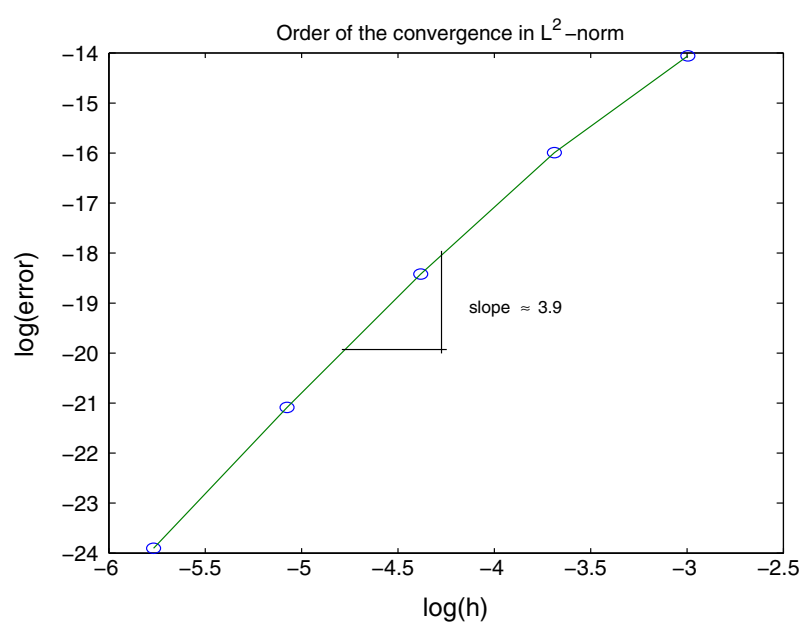

Fig. 1 The $L^{2}$-order of convergence

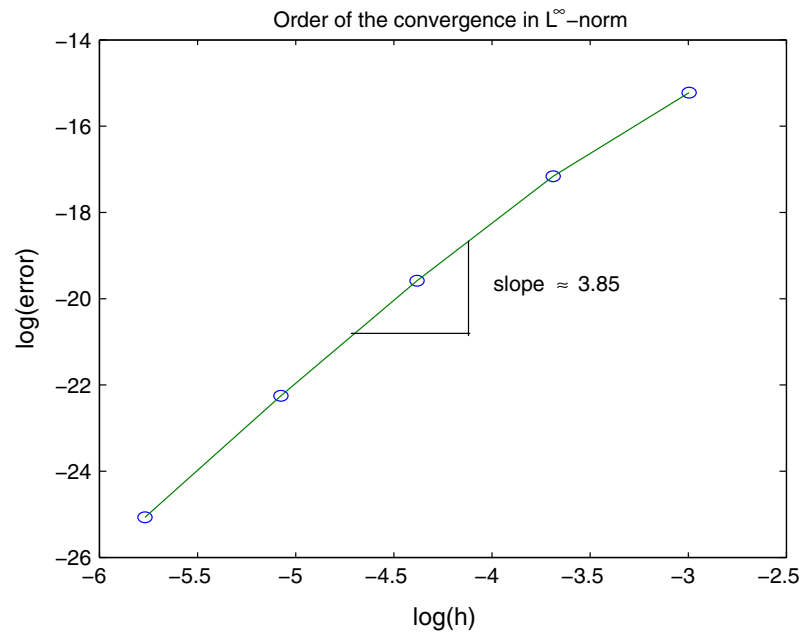

Fig. 2 The $L^{\infty}$-order of convergence

The one dimensional EFK equation is given by

$$
u_{t}+\gamma u_{x x x x}-u_{x x}+f(u)=0, \quad(x, t) \in I \times(0, T]
$$

with initial condition

$$
u(0)=u_{0}=x^{2}(1-x)^{2}, \quad x \in I,
$$

and the boundary conditions

$$
\begin{gathered}
u(0, t)=u(1, t)=0, \quad(x, t) \in \partial I \times(0, T], \\
u_{x}(0, t)=u_{x}(1, t)=0, \quad(x, t) \in \partial I \times(0, T],
\end{gathered}
$$

where $f(u)=u^{3}-u$. 


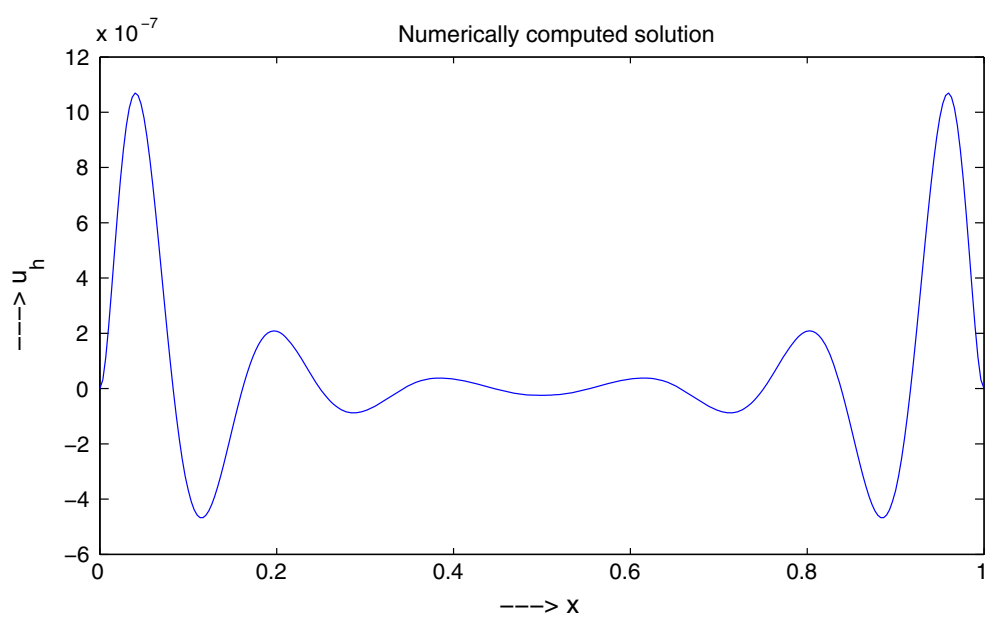

Fig. 3 The numerical solution for $\gamma=1$ and $t=1.0$

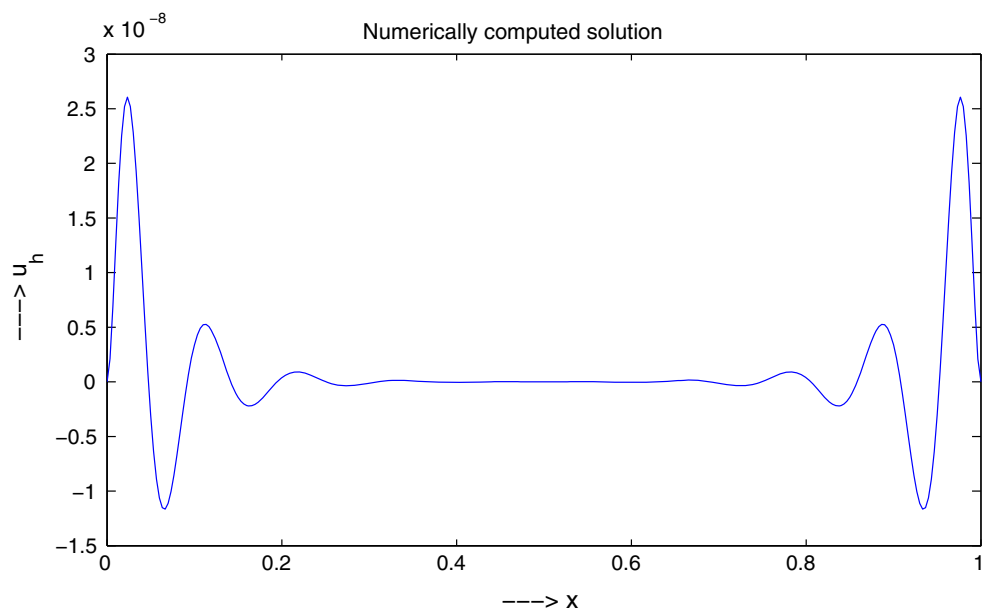

Fig. 4 The numerical solution for $\gamma=0.1$ and $t=1.0$

We divide the domain $I=[0,1]$ into $N_{i}=20,40,80,160,320$ with each of equal intervals $h_{i}$, where

$$
h_{i}=\frac{1}{N_{i}} \quad i=1,2, \ldots, 5 .
$$

For any given positive integer $M$, let $k=\frac{T}{M}$ denotes the size of time discretization and $t_{n}=n k, k=0,1,2, \ldots, M$.

For finding the approximate solution, we use semidiscrete Galerkin method in spatial direction and Crank-Nicolson scheme in temporal direction. Since the exact solution of the EFK equation is not known, Therefore, we have replaced the eact solution by numerical solution $u_{h}$ with $h=1 / 640$. In Table 1 , we show the errors and the optimal order of convergence in tabular form. 


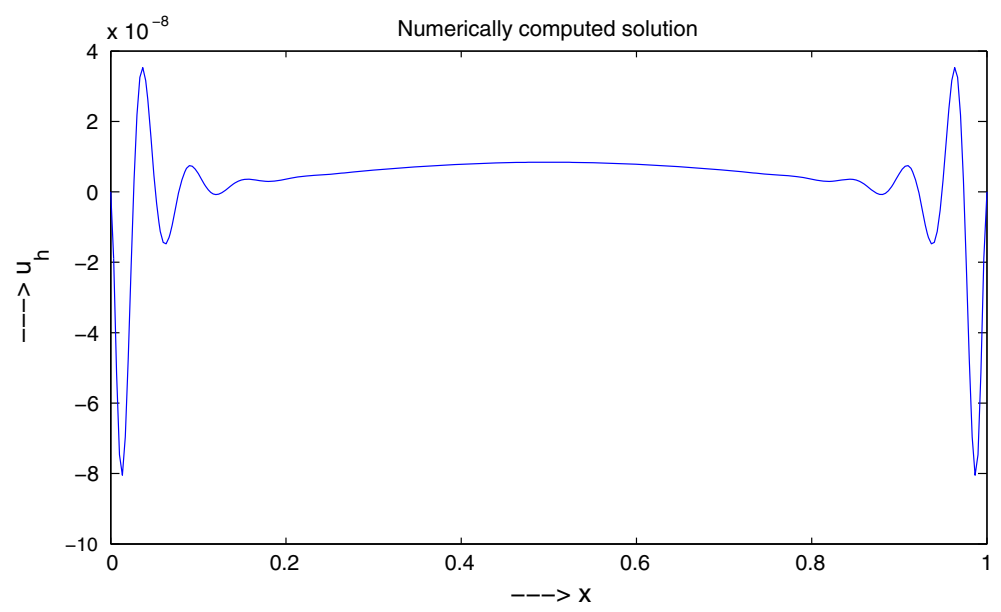

Fig. 5 The numerical solution for $\gamma=0.01$ and $t=1.0$

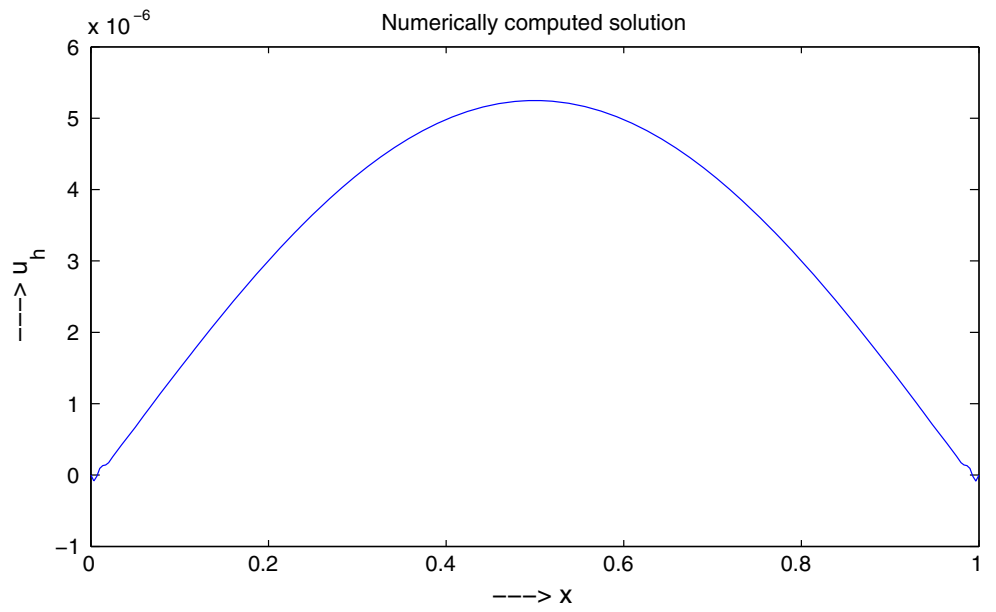

Fig. 6 The numerical solution for $\gamma=0.0001$ and $t=1.0$

Below, we show the graphs Figs. 1 and 2 of order of convergences in $L^{2}$-norm and $L^{\infty}$. norm, respectively. We observe that the numerical order of convergence is equivalent to the theoretical order of convergence. We also show the graphs Figs. 3, 4, 5, 6, 7, and 8 of computed solutions for different values of $\gamma$ and fixed $N=200$. We noticed that as $\gamma$ becomming smaller and smaller the solution profile matches with the Fisher-Kolmogorov equation.

\section{Conclusions}

In this paper, we have studied a fourth order semilinear partial differential equation. We derived a priori bounds using Lyapunov functional and existence uniqueness results are 


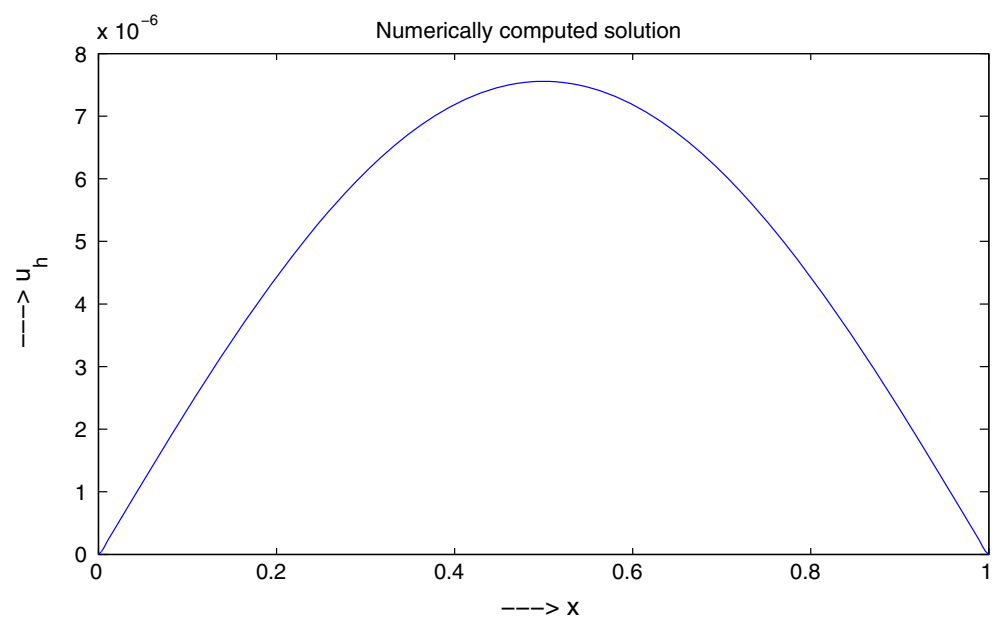

Fig. 7 The numerical solution for $\gamma=0.000001$ and $t=1.0$

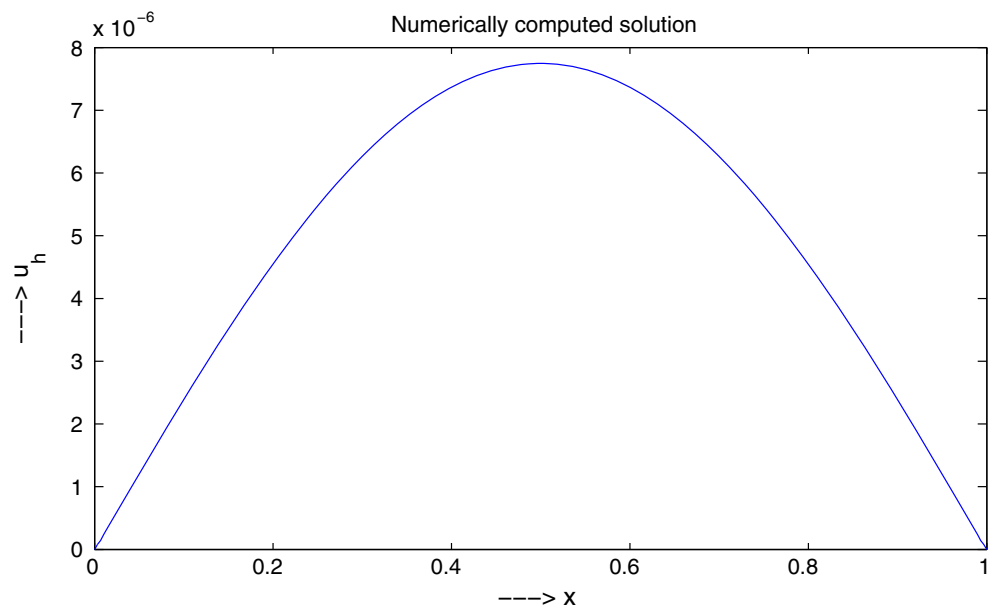

Fig. 8 The numerical solution for $\gamma=0$ and $t=1.0$

discussed. Optimal error estimates are derived in both semidiscrete and fully discrete schemes. Finally, we validated the numerical results with the help of theoretical results.

Acknowledgments The author thanks the referees for their valuable suggestions and comments.

\section{References}

1. Ahlers, G., Cannell, D.S.: Vortex-front propagation in rotating Couette-Taylor flow. Phys. Rev. Lett. 50, 1583-1586 (1983)

2. Aronson, D.G., Weinberger, H.F.: Multidimensional nonlinear diffusion arising in population genetics. Adv. Math. 30, 33-67 (1978)

3. Brenner, S.C., Scott, L.R.: The Mathematical Theory of Finite Element Methods. Springer, New York (2008) 
4. Coullet, P., Elphick, C., Repaux, D.: Nature of spatial chaos. Phys. Rev. Lett. 58, 431-434 (1987)

5. Danumjaya, P., Pani, A.K.: Orthogonal cubic spline collocation method for the extended FisherKolmogorov (EFK) equation. J. Comput. Appl. Math. 174(1), 101-117 (2004)

6. Dee, G.T., van Saarloos, W.: Bistable systems with propagating fronts leading to pattern formation. Phys. Rev. Lett. 60, 2641-2644 (1988)

7. Hornreich, R.M., Luban, M., Shtrikman, S.: Critical behaviour at the onset of $k$-space instability at the $\lambda$ line. Phys. Rev. Lett. 35, 1678-1681 (1975)

8. Jones, L., Nandini, A.P.: An $H^{1}$-Galerkin mixed finite element method for the extended FisherKolmogorov (EFK) equation. Int. J. Numer. Anal. Model., Ser. B 3, 460-485 (2012)

9. Kalies, W.D., Kwapisz, J., VanderVorst, R.C.A.M.: Homotopy classes for stable connections between Hamiltonian saddle-focus equilibria. Commun. Math. Phys. 193, 337-371 (1998)

10. Kesavan, S.: Topics in Functional Analysis and Applications. Wiley Eastern Ltd, New Delhi (1989)

11. Peletier, L.A., Troy, W.C., Vander Vorst, R.C.A.M.: Stationary solutions of a fourth-order nonlinear diffusion equation. Differ. Equ. 31, 327-337 (1995)

12. Peletier, L.A., Troy, W.C.: Chaotic spatial patterns described by the EFK equation. J. Differ. Equ. 129, 458-508 (1996)

13. Peletier, L.A., Troy, W.C.: A topological shooting method and the existence of kinks of the extended Fisher-Kolmogorov equation. Topol. Methods Nonlinear Anal. 6, 331-355 (1996)

14. Qiang, D.U., Nicolaides, R.A.: Numerical analysis of continuum model of phase transition. SIAM J. Numer. Anal. 28, 1310-1322 (1991)

15. van Saarloos, W.: Dynamical velocity selection: marginal stability. Phys. Rev. Lett. 58, 2571-2574 (1987)

16. van Saarloos, W.: Front propagation into unstable states: marginal stability as a dynamical mechanism for velocity selection. Phys. Rev. Lett. A. 37, 211-229 (1988)

17. van Saarloos, W.: Front propagation into unstable states. II. Linear versus nonlinear marginal stability nad rate of convergence. Phys. Rev. Lett. A. 39, 6367-6389 (1989)

18. Zhu, G.: Experiments on director waves in nematic liquid crystals. Phys. Rev. Lett. 49, 1332-1335 (1982) 